\title{
On the relationship between coronary microvascular disease and obesity
}

\author{
Matthieu Pelletier-Galarneau, MD, MSc, ${ }^{\mathrm{a}, \mathrm{b}}$ and Daniel Juneau ${ }^{\mathrm{c,d}}$ \\ a Department of Medical Imaging, Institut de Cardiologie de Montréal, Montreal, QC, Canada \\ b Gordon Center for Medical Imaging, Massachusetts General Hospital, Harvard Medical School, \\ Boston, MA \\ c Department of Medical Imaging, Centre Hospitalier de l'Université de Montréal, Montréal, \\ Canada \\ d University of Ottawa Heart Institute, Ottawa, Canada
}

Received Oct 4, 2021; accepted Oct 4, 2021

doi: $10.1007 / \mathrm{s} 12350-021-02835-z$

\section{See related article, pp. 2434-2445}

Coronary microvascular disease (CMD), also called syndrome $\mathrm{X}$, small vessel disease, or nonobstructive coronary heart disease, is an increasingly recognized cause of chest pain in patients with nonobstructive coronary artery disease (CAD), especially in younger women. ${ }^{1,2}$ CMD shares several risk factors with CAD and may precedes development of obstructive epicardial CAD. Identification of patients suffering from CMD is important as they are at increased risk of adverse events and may benefit from more intensive medical therapy and lifestyle changes. Over the past decade, there has been a rising interest in the medical community about CMD. The democratization of positron emission tomography (PET) myocardial perfusion imaging (MPI), with it's ability to non-invasively quantify absolute myocardial blood flow (MBF) and flow reserve (MFR), is no stranger to this growing attention. ${ }^{3}$ Indeed, under maximal coronary vasodilation and in the absence of significant epicardial stenosis, hyperemic MBF and MFR are proxies of the microvascular function.

In their study, Wang et $\mathrm{al}^{4}$ evaluated the relationship between adiposity distribution and measurements of MBF and MFR using ${ }^{13} \mathrm{~N}$-ammonia PET MPI. They prospectively recruited 128 symptomatic patients

\footnotetext{
Reprint requests: Matthieu Pelletier-Galarneau, MD, MSc, Department of Medical Imaging, Institut de Cardiologie de Montréal, Montreal, QC H1T 1C8, Canada; mattgalarneau@gmail.com J Nucl Cardiol 2022;29:2446-7. $1071-3581 / \$ 34.00$

Copyright (c) 2021 American Society of Nuclear Cardiology.
}

without obstructive epicardial CAD who underwent a PET MPI study. They subdivided their study population based on waist circumference (WC) and body mass index (BMI). They observed that central obesity, defined as $\mathrm{WC}>95 \mathrm{~cm}$ in men and $>90 \mathrm{~cm}$ in women, was associated with lower hyperemic MBF and MFR, and higher prevalence of CMD. Interestingly, they did not observe such association with excess weight alone, defined as BMI $>25 \mathrm{~kg} / \mathrm{m}^{2}$ with normal WC, supporting the concept that different metabolic profiles, or phenotypes, can be found among obese patients. This result is not surprising as BMI has long been recognized as an imperfect marker of metabolic health. Although BMI correlates with body fat, there is substantial variability in metabolic dysfunction for any given $\mathrm{BMI},{ }^{5}$ giving rise to the controversial concept of "metabolically healthy" obesity. Metabolically healthy obesity, which represents approximately $35 \%$ of all obese individuals, generally refers to individuals without definite cardiometabolic disorder, including insulin resistance, hypertension, etc. ${ }^{5}$ Interestingly, among obese individuals, those categorized as metabolically healthy have lower risk of cardiovascular diseases compared to the "metabolically unhealthy", but are still at higher risk compared to metabolically healthy lean individuals., Different phenotypes of obesity have also been described based on CMD, with studies showing that MFR is superior to BMI to predict cardiovascular adverse events. ${ }^{7}$ The results of the study by Wang et al. further support this notion by showing that subjects with excess weight (elevated BMI) and no central obesity (normal WC) have higher hyperemic MBF and MFR compared to those with central obesity and no excess weight, but lower hyperemic MBF and MFR compared to those with normal weight and without central obesity. Furthermore, 
the subjects with normal weight and central obesity had comparable hyperemic MBF and MFR compared to those with both excess weight and central obesity.

PET is emerging as the non-invasive modality of choice for the evaluation of patients with suspected CMD. ${ }^{2}$ However, there are currently no universally accepted threshold for hyperemic MBF or MFR, as technical factors such as choice of radiotracers and stress agents may affect such threshold. In the current study, a hyperemic $\mathrm{MBF}<2.3 \mathrm{~mL} / \mathrm{g} / \mathrm{min}$ and MFR $<2.5$ were used as thresholds, which is comparable to other studies using ${ }^{13} \mathrm{~N}$-ammonia PET although lower thresholds are sometime used. Thresholds of 2.0 to 2.5 have been proposed by the Coronary Vasomotion Disorders International Study (COVADIS) group, acknowledging methodology-related differences. ${ }^{8}$ In this study, CMD was defined by the presence of either abnormally low hyperemic MBF or MFR. It is worth noting that there is currently no consensus on the best criteria to confirm the presence of CMD on PET and that the author's definition of CMD may suffer from suboptimal specificity. For instance, elevated resting flow with normal hyperemic MBF may result in low MFR, which may in fact represent normal hyperemic response in otherwise healthy individuals. Likewise, mildly reduced hyperemic flows with normal MFR is often seen in the absence of significant coronary disease, such as in patients treated with beta-blockers.

Interpretation of the study results must also take into consideration that some patients underwent adenosine stress while others received dipyridamole. Although the authors cite articles reporting similar hyperemic flows with the two agents, other studies reported higher hyperemic flows and lower coronary resistance with adenosine..$^{9,10}$ The differences in hyperemic MBF and MFR observed in the different groups could be partially attributed to the different proportion of patients undergoing adenosine vs dipyridamole, although it is unlikely that this alone accounts for the differences. Ideally, all subjects would have undergone identical stress protocols.

Despite these minor limitations, the results of this study help to better understand the intricate relationship between obesity and CMD. They suggest that PET could potentially be used to further refine the concept of metabolically healthy obesity and identify the subgroup of obese patients who are at higher risk of adverse events and who may need more aggressive therapy. Further prospective studies including outcome data are needed to better delineate these potential roles of PET.

\section{Disclosures}

$D . J$. has received consultant fees from AbbVie, Pfizer and Advanced Accelerator Applications. M.P-G. is supported by a Junior 1 Research Award from the Fonds de Recherche du Québec - Santé (FRQS). M.P-G. has received consultant fees and research funds from Jubilant Radiopharma.

\section{References}

1. Safdar B, D’Onofrio G, Dziura J, Russell RR, Johnson C, Sinusas AJ. Prevalence and characteristics of coronary microvascular dysfunction among chest pain patients in the emergency department. Eur Heart J Acute Cardiovasc Care 2020;9:5-13. https://doi. org/10.1177/2048872618764418.

2. Pelletier-Galarneau M, Dilsizian V. Microvascular angina diagnosed by absolute PET myocardial blood flow quantification. Curr Cardiol Rep 2020;22:9. https://doi.org/10.1007/s11886-020-12612.

3. Pelletier-Galarneau M, Martineau P, El Fakhri G. Quantification of PET myocardial blood flow. Curr Cardiol Rep 2019;21:11. h ttps://doi.org/10.1007/s11886-019-1096-X.

4. Wang R, Li X, Huangfu S, Yao Q, Wu P, Wu Z, et al. Combining body mass index with waist circumference to assess coronary microvascular function in patients with non-obstructive coronary artery disease. J Nucl Cardiol 2021. https://doi.org/10.1007/s123 50-021-02788-35.

5. Magkos F. Metabolically healthy obesity: What's in a name? Am J Clin Nutr 2019;110:533-9. https://doi.org/10.1093/ajcn/nqz133.

6. Eckel N, Meidtner K, Kalle-Uhlmann T, Stefan N, Schulze MB. Metabolically healthy obesity and cardiovascular events: A systematic review and meta-analysis. Eur J Prev Cardiol 2016;23:956-66. https://doi.org/10.1177/2047487315623884.

7. Bajaj NS, Osborne MT, Gupta A, Tavakkoli A, Bravo PE, Vita T, et al. Coronary microvascular dysfunction and cardiovascular risk in obese patients. J Am Coll Cardiol 2018;72:707-17. https://doi. org/10.1016/j.jacc.2018.05.049.

8. Ong P, Camici PG, Beltrame JF, Crea F, Shimokawa H, Sechtem $\mathrm{U}$, et al. International standardization of diagnostic criteria for microvascular angina. Int J Cardiol 2018;250:16-20. https://doi. org/10.1016/j.ijcard.2017.08.068.

9. Mamede M, Tadamura E, Hosokawa R, Ohba M, Kubo S, Yamamuro $\mathrm{M}$, et al. Comparison of myocardial blood flow induced by adenosine triphosphate and dipyridamole in patients with coronary artery disease. Ann Nucl Med 2005;19:711-7.

10. Rossen JD, Quillen JE, Lopez AG, Stenberg RG, Talman CL, Winniford MD. Comparison of coronary vasodilation with intravenous dipyridamole and adenosine. J Am Coll Cardiol 1991;18:485-91. https://doi.org/10.1016/0735-1097(91)90604-8.

Publisher's Note Springer Nature remains neutral with regard to jurisdictional claims in published maps and institutional affiliations. 